\title{
High Energy Particles from the Universe
}

\author{
Rene A. Ong \\ Enrico Fermi Institute \\ University of Chicago, Chicago, IL 60637
}

\section{Outline}

It is an exciting time to be working at the interface between physics and astronomy. Experiments built to detect astrophysical and atmospheric sources of neutrinos, such as Super-Kamiokande, are providing what may be the first definitive evidence for neutrino mass. Cosmology experiments, such as those measuring the the redshifts of type Ia supernovae and those determining the anisotropy of the microwave background, may have an important impact on particle physics as they confront our general view of the origin of the Universe. There have been similarly exciting developments at the high energy frontier of astronomy. Among other things:

1. we have detected a gamma-ray burst that is probably the most powerful explosion recorded since the Big Bang,

2. we have discovered extragalactic astrophysical sources that beam intense fluxes of $\mathrm{TeV} \gamma$-radiation to us, and

3. we have observed individual particles (possibly protons) arriving from outer space with energies exceeding 25 Joules.

These new developments are clearly of prime importance for the field of high energy astrophysics, but, even more, research in this area in the future may make important contributions to particle physics depending, of course, on the nature of the discoveries.

In this paper, I provide a summary of the field of high-energy astronomy using photons, cosmic rays, and neutrinos. This field is rapidly developing because of recent discoveries and because of new experimental techniques that derive largely from accelerator-based particle physics detectors. I start with a broad overview of the field and discuss the general scientific motivations. Then, the three exciting developments listed above are described in more detail, followed by a discussion of the theoretical considerations. A selective review of the field in terms of the experimental techniques, results, and future prospects makes up the last part of the paper. I conclude with a summary of the prospects for the future. 


\section{Broad Overview}

We learn about the Universe outside the immediate neighborhood of the Solar System by studying the arrival of four distinct messengers: 1) photons, 2) cosmic rays, 3) neutrinos, and 4) gravity waves [1]. Here, cosmic rays are defined as nuclei (p, n, $\mathrm{He}^{++}$, etc.) electrons, and their antiparticles. In the future, we may discover other stable particles that convey information across interstellar space. If we do, we can add them to the previous list.

To date, the detection the photons over a wide range of energies has been the basis for the vast majority of astronomical discoveries. Cosmic rays provide important information about high energy processes occurring in our galaxy. Neutrinos and gravity waves both offer great astronomical potential, but are difficult to detect. So far, the neutrino source list is limited to our Sun and the supernova SN1987A.

Photon, cosmic ray, and neutrino astronomy are closely related at high energies $(\mathrm{E}>1 \mathrm{GeV})$, both in terms of their astrophysical production mechanisms and in terms of their detection techniques. High energy particles are produced astrophysically by acceleration processes rather than by thermal processes which dominate at lower energies. The high energy particle fluxes typically exhibit rapidly falling power-law spectra, which leads to the requirement of very large detectors. The high energy domain spans a wide dynamic range (from $1 \mathrm{GeV}$ to $10^{11} \mathrm{GeV}$ ), and we cannot expect a single detection technique to work at all energies.

In the future, we imagine studying astrophysical sources with multiple messengers that provide complementary information. A prototypical source would be gamma-ray bursts (GRBs). We know a great deal about GRBs from the electromagnetic radiation they produce, but there is also speculation that they are the source of the highest energy cosmic rays [2] and that they produce a detectable neutrino flux [3]. Thus, we anticipate that astronomy using these different messengers will become interrelated as the experiments become more powerful and as more detections are made.

The general scientific motivation for particle astronomy is multi-faceted. On the physical side, we use high energy radiation to probe extreme conditions of magnetic or gravitational potential. Our understanding of such astrophysical situations is still far from complete. The copious flux of high energy cosmic rays argues for efficient astrophysical accelerators with beam energies well beyond what we can achieve on Earth. High energy particle astronomy may also shed light on aspects of particle physics or cosmology beyond their respective standard models. On the astronomical side, the movement towards high energies continues the historical expansion of astronomy from optical light into new wavebands (radio, infrared, X-ray, and $\gamma$-ray). It is important to emphasize, however, that when discussing the many aspects of high

energy astronomy, there is not yet a generally applicable "Standard Model". For the experimentalist, this situation is ideal in that there are few constraints. New experimental results often change the general paradigm. 


\section{Three Recent Exciting Results}

Here I discuss three of the most exciting results in this field in the last few years. The choices are, of course, very subjective.

\subsection{Gamma Ray Bursts}

Even after thirty years of research, the nature of gamma ray bursts (GRBs) remains one of the most important mysteries of astrophysics. We know from the BATSE detector on the Compton Gamma Ray Observatory that the arrival directions of GRBs are consistent with isotropy [1. However, the relatively poor angular resolution of BATSE of a few degrees has made the detection of GRB counterparts (i.e. sources at optical or radio wavelengths) difficult. The lack of counterparts has hindered efforts to pin down the distance scale of GRBs.

In 1997, a major breakthrough was achieved with the first detection of optical counterparts. This work was accomplished by the Beppo/SAX satellite detector in conjunction with powerful optical telescopes, both on the ground (e.g. Keck) and in space (e.g. HST). By using a combination of wide and narrow-field X-ray telescopes, Beppo/SAX determines the positions of some bursts with an accuracy of several arc minutes. This excellent localization allowed the detection of fading optical counterparts for several dozen bursts [5]. Redshift values have been determined for approximately twenty counterparts; they indicate that the correlated bursts are cosmological in origin (typical $z \sim 1$ ). The typical inferred energy outputs of the bursts range between $10^{51}$ and $10^{53}$ ergs (assuming isotropic emission).

The detection of optical counterparts to GRBs is clearly a landmark discovery. Until 1999, however, optical observations were limited to time periods well after the actual bursts because of the long delay (typically 8 hours) required by Beppo/SAX to achieve accurate position localization. What we really want to do is to carry out out simultaneous $\gamma$-ray and optical observations of GRBs. Such observations require a fast slewing telescope with a wide field of view to cover the large BATSE error box.

Several wide-field optical telescopes have been constructed or are currently under

development. One of the pioneering experiments, known as ROTSE-I, consists of an array of $35 \mathrm{~mm}$ telephoto lenses coupled to large format CCD detectors. With an overall field of view of $16^{\circ} \times 16^{\circ}$, and by slewing in an automated fashion upon receiving an alert from the GRB Coordinates Network (GCN), ROTSE is ideally suited for rapid optical follow-up of GRBs. The careful design of the experiment paid off on January 23, 1999 when ROTSE made the first detection of contemporaneous optical radiation from a GRB [9].

From the perspective of BATSE, GRB990123 was relatively ordinary, but the optical signature detected by ROTSE, was truly remarkable. As shown in Figure 3.1, the optical brightness increased by 3 magnitudes in 25 seconds and then waned by 5 

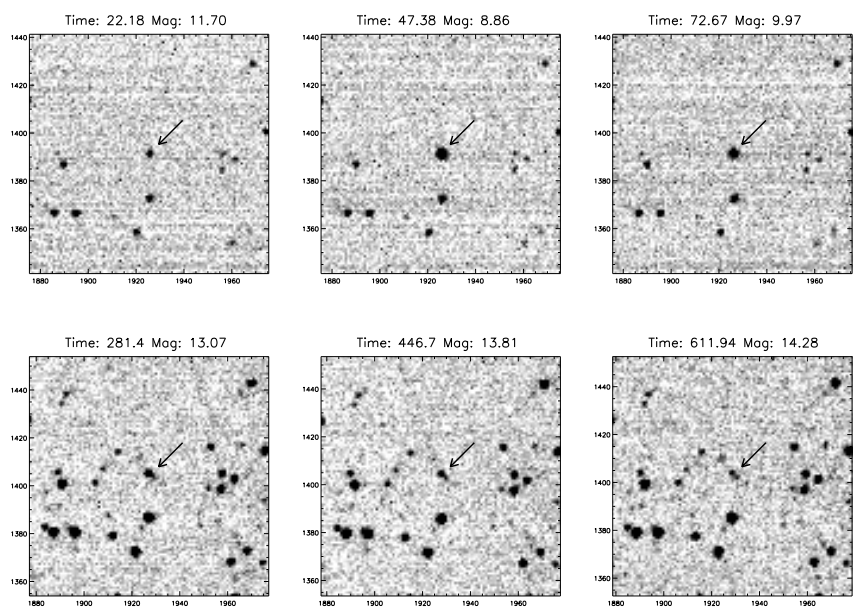

Figure 1: Detection of powerful optical radiation from a gamma ray burst in progress [9]. Six successive CCD images taken by the ROTSE-I experiment are shown for GRB990123. At a redshift of 1.60 and a peak magnitude of 8.95, this burst was the most luminous astronomical object ever detected.

magnitudes over a period of 8 minutes. At its peak brightness, the optical magnitude was 8.95 which, when combined with the measured redshift value of 1.60 , meant that this burst was the most luminous object ever detected. GRB990123 was brighter than the brightest quasar by several orders of magnitude. Assuming isotropic emission, the inferred energy release of GRB990123 exceeds $10^{54}$ ergs. Theoretical models, already grappling to explain the wide variety of GRB phenomena, are further strained to deal with this remarkable energy output.

A wide range of theoretical models have been proposed to explain gamma ray bursts. There are almost as many models as bursts! The basic difficulty is to construct a physical mechanism that can produce and extract the intense high energy emission we observe. The general picture calls for a cataclysmic event which produces a relativistic fireball of material with Lorentz factors approaching 1000 [6]. The relativistic material escapes from the region of high energy density along a collimated jet. High energy radiation results when the jet collides with nearby ambient material. The nature of the original cataclysmic event is not fully understood. Generally favored pictures include colliding neutron stars and hypernovae ("failed supernovae" of heavy stars) [7].

The recent discoveries in the area of gamma ray bursts are clearly profound, but the overall puzzle is far from being solved. Many additional questions remain. For example, when considering the time durations and spectral shapes of GRBs, we know 
from the BATSE data that there are at least two classes of bursts, (if not more) [8]. For instrumental reasons, Beppo/SAX is sensitive only to longer bursts (duration greater than 1 second). Thus, so far, we can confidently ascribe a cosmological origin only to a portion of the burst population. The medium and short length bursts may have a different origin. We can expect GRB research to remain exciting for years to come.

\section{2 $\mathrm{TeV} \gamma$-rays from Extragalactic Sources}

The field of very high energy (VHE) $\gamma$-ray astronomy has come of age in the last ten years [10, 11]. Ground-based telescopes using the atmospheric Cherenkov technique have made strong detections of $\mathrm{TeV}$ photons from galactic and extragalactic sources. The most exciting development in this area has been the discovery of strong VHE emission from active galactic nuclei (AGN), which make up a broad class of extragalactic objects including quasars. AGN are powerful sources at all wavelengths, but the importance of their $\gamma$-ray output has only recently been fully appreciated. We now know that in some AGN, those known as blazars, the bulk of their power is emitted at high energies. Explaining this observational fact is an important problem facing theorists.

In 1997, the blazar source Markarian 501 (Mrk 501) entered a period of dramatic activity. The TeV $\gamma$-ray emission from the source increased by up to a factor of fifty from earlier epochs. Figure 3.2 shows the VHE $\gamma$-ray flux detected from this source over a four year period by the Whipple experiment. The difference between the average flux level seen in 1997 and in the other years is striking. At certain times, the $\gamma$-ray flux from Mrk 501 exceeded the brightest known source in our galaxy, the Crab Nebula, by factors of three to four. This happened in spite of the fact that Mrk 501 is more distant from us than the Crab by a factor of 10,000. At its maximum brightness, Mrk 501 beamed $10^{11}$ VHE photons per second to the Earth's surface. The VHE spectrum extends to maximum energies above $20 \mathrm{TeV}$.

Another impressive feature of the 1997 emission from Mrk 501 was the high degree of variability observed. The $\gamma$-ray flux varied by as much as an order of magnitude from night to night and by factors of two on hourly time scales. These variations imply that the acceleration region where the $\gamma$-rays are produced must be very compact, presumably several light hours across (times any relativistic Doppler factors). The flux variations of Mrk 501 were studied by several state-of-the-art Cherenkov telescopes around the globe and there was good agreement between the flux levels recorded by the different experiments.

AGN are such luminous objects that the only feasible source for their power is in the intense gravitational field near black holes. The general picture of an AGN is a supermassive $\left(10^{8}-10^{9} \mathrm{M}_{\odot}\right)$ black hole surrounded by a rotating disk. Accreting material provides the power for the broad-band emission observed. It also powers 


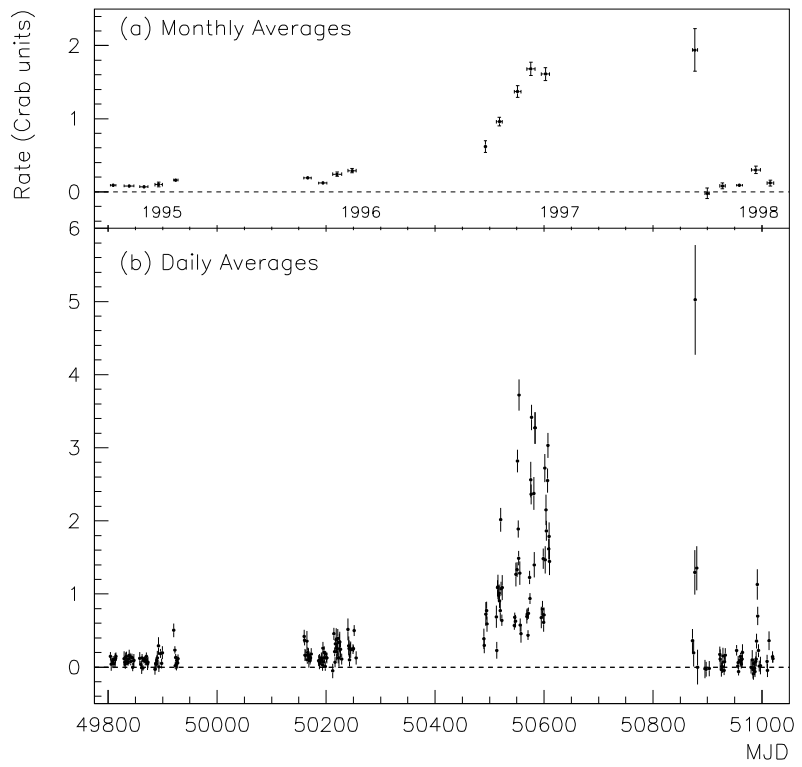

Figure 2: The flux of very high energy $\gamma$-rays from the extragalactic blazar source Markarian 501 [12]. The flux over a four year period as recorded by the Whipple Observatory is shown normalized by the flux from the Crab Nebula (the brightest known galactic source). In 1997, dramatic increases in the flux level and in the degree of variability were recorded. The $\gamma$-ray energies range between $250 \mathrm{GeV}$ and $20 \mathrm{TeV}$.

relativistic jets directed along the angular momentum axis of the black hole. As shown in Figure 3.2, shock acceleration in the jets produces high energy beams of electrons or protons which interact with radiation fields to produce secondary beams of $\gamma$-rays and neutrinos. A key aspect of this model is that we observe blazars when their beams are directed into our line of sight.

In the general blazar picture, the high energy $\gamma$-rays probe acceleration processes in the jet. The fact that very rapid $\gamma$-ray variability has been observed indicates that the acceleration region relevant to high energy particle production is deep within the jet, and possibly quite close to the black hole itself. Thus, understanding the VHE $\gamma$-ray emission is of crucial interest.

\subsection{Cosmic Rays with Energies Exceeding $10^{20} \mathbf{e V}$}

We have known about the existence of extremely high energy $\left(E>10^{20} \mathrm{eV}\right)$ cosmic rays for more than forty years. In 1966, it was realized by Greisen, Zatsepin, and Kuz'min (GZK) [14] that cosmic rays above the energy of $\sim 6 \times 10^{19} \mathrm{eV}$ (if they ex- 


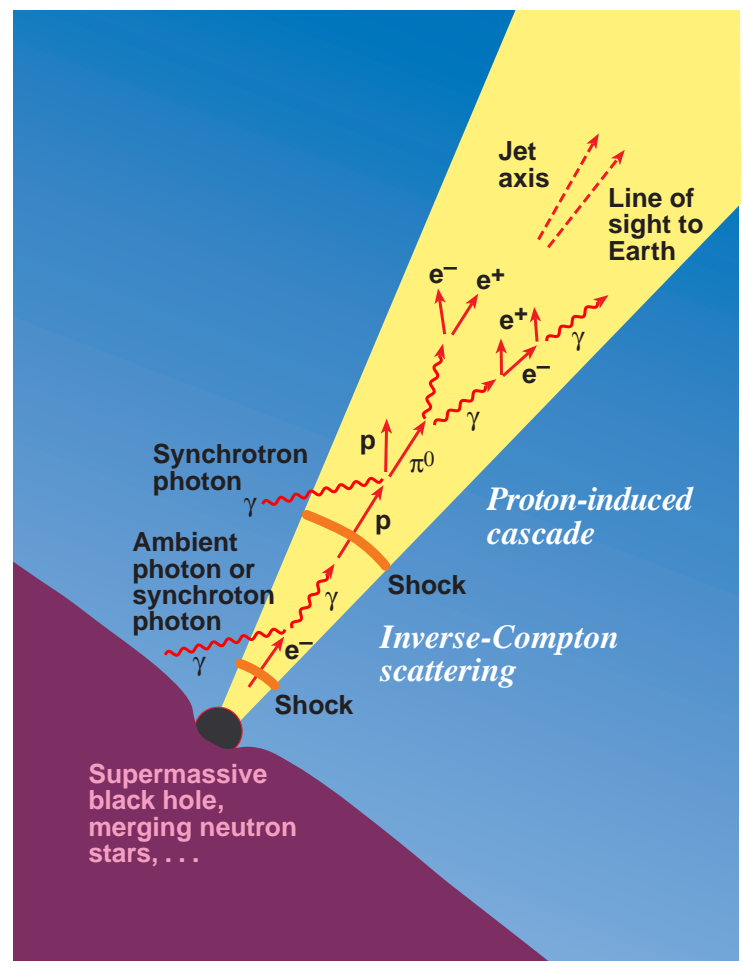

Figure 3: Artist's conception of the acceleration processes in a blazar 13. Black hole accretion powers relativistic jets of material. In the jets, electrons or protons are accelerated to high energies via shocks. High energy $\gamma$-rays are produced from the inverse-Compton scattering of electrons or from cascades initiated by protons. We detect emission from those sources which are beamed into our line of sight.

isted!) would interact with the $3 \mathrm{~K}$ cosmic microwave background radiation (CMBR). This GZK cutoff limits the mean free path for the highest energy cosmic rays to less than $100 \mathrm{Mpc}$, a distance that is quite small in comparison to typical extragalactic scales. Until recently, conventional wisdom held that cosmic rays above the GZK cutoff would not be detected on Earth because: 1) it was hard to construct astrophysical sources capable of accelerating particles to these energies, and 2) if such source existed, they would almost certainly be located at great distances and the cosmic rays they produced would be absorbed by the CMBR. This wisdom proved to be wrong. It now seems clear that the cosmic ray spectrum continues to energies of $10^{20} \mathrm{eV}$, and beyond.

The most compelling evidence for particles beyond the GZK cutoff comes from the AGASA experiment [15]. AGASA is a large surface array covering an area of approximately $100 \mathrm{~km}^{2}$, and located in the central portion of the Japanese island of 
Honshu. The array samples the particle cascade in giant air showers produced from extremely high energy cosmic ray interactions in the atmosphere. The cosmic ray energy is estimated from the particle density determined a fixed distance from the core of the shower. Uncertainties in the energy measurement are estimated by several techniques, including simulation methods. The typical energy resolution is $\sim 30 \%$. More importantly, the proportion of events with a $50 \%$ or more overestimation in energy is less than $3 \%$.

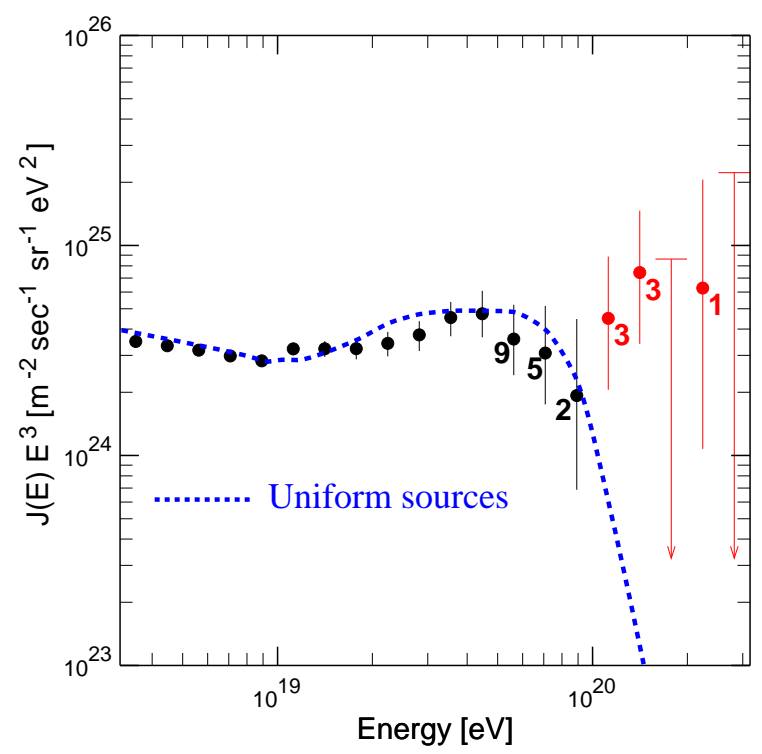

Figure 4: Energy spectrum of the highest energy cosmic rays measured by the AGASA experiment [15]. As indicated, seven events are detected above $10^{20} \mathrm{eV}$. The dashed line corresponds to the spectrum expected from a population of extragalactic sources distributed uniformly in the Universe. For display purposes, the differential particle flux is multiplied by the energy cubed.

The latest cosmic ray energy spectrum released by AGASA is shown in Figure 3.3 [15]. Based on a data sample collected between 1993 and 1998, the spectrum shows no evidence for a cutoff above $6 \times 10^{19} \mathrm{eV}$. Instead, seven events are detected above $10^{20} \mathrm{eV}$. The measured spectrum from AGASA is inconsistent with that expected from a population of extragalactic sources distributed uniformly in the Universe. Barring an unforeseen loophole to the GZK cutoff, the AGASA results, in conjunction with the detection by the Fly's Eye experiment of a cosmic ray at $3 \times 10^{20} \mathrm{eV}$ [16], strongly support the idea the sources of the highest energy cosmic ray events are "local".

Considering the enormous rigidity of $10^{20} \mathrm{eV}$ particles and the weakness of the intergalactic magnetic field $\left(B<10^{-10} \mathrm{~g}\right)$, we do not expect substantial deflection of such particles over path lengths of $50 \mathrm{Mpc}$. Thus, it is reasonable to consider doing 
astronomy with the extremely high energy cosmic rays. The arrival directions of the cosmic rays above $10^{19} \mathrm{eV}$ have been examined. No obvious concentration in the sky can be discerned. Also, there is no significant correlation between the cosmic ray positions and those of known astronomical sources (e.g. quasars, radio galaxies, etc.). Thus, we are faced with a quandary similar to that of gamma ray bursts. Since the sources of the highest energy cosmic rays cannot be correlated with known astronomical objects, they must represent something new. To achieve their extreme energies, the cosmic rays must be produced in a truly remarkable astrophysical accelerator. They could, in fact, come from physics at a higher mass scale. It is perhaps this latter possibility that makes these particles so intriguing.

\section{Theoretical Considerations}

\subsection{Extreme Astrophysics}

As introduced in Section 2, we expect high energy $\gamma$-rays, cosmic rays, and neutrinos to be produced via particle interactions at sites of powerful acceleration. When discussing such production, we must distinguish between the power source and the acceleration mechanism. For sources using conventional (i.e. known) physics, the power source will make use of extreme electromagnetic or gravitational potentials. Pulsars, such as the Crab, are examples of the former case. Supernova remnants and AGN are examples of the latter.

High energy $\gamma$-radiation has been detected from a number of known radio pulsars. The radiation can be categorized as being pulsed (i.e. with the same period as that detected in the radio) or unpulsed. For both categories, the ultimate power source derives from the spin-down of a highly magnetized neutron star. At the highest energies, the radiation is believed to originate from inverse-Compton scattering of soft photons by a relativistic wind of electrons [17]. In the case of the Crab, from which $\gamma$-rays up to $50 \mathrm{TeV}$ have been detected, the electron spectrum is believed to extend above $1 \mathrm{PeV}$, making the Crab the high-energy accelerator known in the Universe.

Supernova remnants (SNRs) are attractive candidates for particle acceleration because of the enormous power contained in the original explosion. Supernova explosions typically release $10^{51}$ ergs of kinetic energy and they occur every few decades somewhere in the galaxy. Therefore, the average power injected into the interstellar medium by these explosions is $\sim 10^{42} \mathrm{ergs} \mathrm{s}^{-1}$. Since the power required to replenish the cosmic rays is $10^{40}-10^{41} \mathrm{ergs} \mathrm{s}^{-1}$, supernova remnants can explain the origin of the cosmic rays if $1-10 \%$ of the kinetic energy released in the explosion goes into the acceleration of protons and nuclei.

Particle acceleration in SNRs is expected to occur at the shock front produced as the remnant material traverses the interstellar medium. Charged particles scatter 
off magnetic field irregularities as they diffuse back and forth across the shock front. Because the velocity distributions of the scattering centers on either side of the shock are isotropic, the particles see a converging flow of scattering centers on both sides of the front. They thus gain energy with each round trip passage. This particle acceleration process, first proposed by Fermi in a different context [18], continues as long as the particles are contained in the vicinity of the shock front. The containment time is proportional to energy, and so diffusive shock acceleration naturally leads to a power law energy spectrum for the accelerated particles, $\left(\mathrm{dN} / \mathrm{dE} \propto \mathrm{E}^{\alpha}\right)$. For strong (highly supersonic) shocks, the power law spectral index is $\alpha \sim-2$.

The maximum obtainable particle energy in SNRs is determined by the lifetime of the shock itself. As the shock wave expands, it slows downs and weakens. For typical shock lifetimes ( $~ 5000 \mathrm{yr})$, the maximum particle energy is calculated to be $\mathrm{Z} \times 10^{14} \mathrm{eV}$, where $\mathrm{Z}$ is the particle charge [19]. Thus, SNRs offer a plausible explanation for the origin of cosmic rays up to an energy of $10^{15} \mathrm{eV}$ (and possibly up to $10^{16} \mathrm{eV}$ ), but a new source is required to explain higher energy cosmic rays.

There is a great deal of speculation about the origin of particles above $10^{16} \mathrm{eV}$. Galactic objects do not in general have the combination of size and magnetic field strength to contain a particle at these energies [20]. Therefore, it is generally believed that the sources must be extragalactic. AGN are attractive possibilities because they are known to be powerful emitters of $\gamma$-rays. They may also produce very high energy cosmic rays and neutrinos.

The general model for AGN was outlined in Section 3.2 (see Figure 3.2). Charged particles are accelerated to high energies in the jet via the Fermi shock mechanism. The accelerated particles are likely to be electrons or protons (or a combination of the two). Electrons are certainly needed to explain the broad-band synchrotron emission observed. If electrons dominate, $\gamma$-rays could naturally be produced by inverseCompton scattering off low energy photons [21]. The soft photons may originate from synchrotron radiation, from disk emission, or from reprocessed emission from clouds or dust [22]. If protons dominate, they would interact with radiation fields to produce electromagnetic cascades that would ultimately produce high energy $\gamma$-rays [23]. Protons may also produce neutrinos via a beam dump mechanism (i.e. by impinging on material to produce charged pions which then decay).

Extragalactic sources, such as AGN, may explain the origin of cosmic rays between $10^{16} \mathrm{eV}$ and $10^{19} \mathrm{eV}$. However, as discussed in Section 3.3, the highest energy particles cannot come from large extragalactic distances. Their origin is a true mystery. Given our inability to explain their origin via known astrophysics, we are forced to seriously consider other possibilities, such as new astrophysics or particle physics beyond the Standard Model. 


\subsection{Non-Standard Astrophysics or Particle Physics}

When explaining the existence of particles arriving at Earth with very high energies, we must naturally consider possible sources outside known astrophysics. In this case, particles would acquire their energies in a "top down" picture,i.e. as coming from physics at a higher mass scale, as opposed to the "bottom up" picture of acceleration. Cosmology must come into play here, because the early Universe offers the most natural conditions needed to generate interactions or particles beyond the Standard Model. Here we give a few examples of non-standard sources of very high energy particles.

There is speculation that the highest energy cosmic rays result from the collapse of topological defects produced in the early Universe [24]. Defects such as cosmic strings resulting from phase transitions could produce grand unified (GUT) scale particles at with masses of $10^{14}-10^{15} \mathrm{GeV}$. GUT particles would decay to leptons and quarks, producing electromagnetic and hadronic cascades whose eventual tertiary products would cosmic rays or $\gamma$-rays. Under certain assumptions, the cosmic rays would be produced close enough to Earth to avoid the GZK cutoff.

Another interesting possibility is that of primordial black holes. Black holes are not truly black, but emit a spectrum of radiation [25]. As the black hole loses energy, it gets hotter, and this results in more available quantum states for the emission. Eventually, the black hole evaporates in a final explosion that releases a burst of radiation. Small black holes created in the early Universe with masses near $10^{14} \mathrm{~g}$ would be in the process of evaporation now. Such primordial black holes would produce bursts of VHE $\gamma$-rays on time scales of microseconds to seconds [26].

The search for evidence of supersymmetry is a very important frontier area of particle physics. Given the possibility that supersymmetry may manifest itself at $\mathrm{TeV}$ energies, it is natural to consider VHE astrophysical signatures. One possible signature would be the direct detection of neutral supersymmetric particles. For example, it has been proposed that the highest energy cosmic rays are supersymmetric strongly interacting particles $\left(S^{0}\right)$ having a much greater path length through the CMBR than ordinary nucleons [27]. A second possible signature for supersymmetry would be the detection of secondary decay products from a supersymmetric particle. The most interesting possibility here is that of the neutralino, $\tilde{\mathrm{X}}^{0}$. Neutralinos may be the lightest supersymmetric particles and they may also comprise a large fraction of the dark matter in the galaxy [28]. Since neutralinos must couple to ordinary matter through weak interactions, the weak scale cross-section determines the temperature at which the neutralino would freeze out, and hence its relic abundance. Neutralinos concentrating in the galactic center would annihilate via loop processes, $\tilde{\mathrm{X}}^{0} \tilde{\mathrm{X}}^{0} \rightarrow$ $\gamma \gamma, Z \gamma$, yielding a very high energy $\gamma$-ray signature [29]. Similarly, neutrinos would be produced from the annihilations of gravitationally trapped neutralinos in the center of the Sun or the Earth. Estimates for the $\gamma$-ray or neutrino fluxes are sensitive to 
parameters of the given model of supersymmetry, to the mass of the neutralino, and to the density profile of galactic dark matter. In spite of large uncertainties in expected flux levels, the search for supersymmetry by these techniques is important for several reasons: 1) the signature, a highly directional, mono-energetic flux of $\gamma$-rays or neutrinos, would be unambiguous, (i.e. it would provide "smoking gun" evidence for supersymmetry), 2) a statistically significant detection would allow measurement of the neutralino mass, and 3) the next generation of $\gamma$-ray and neutrino telescopes are sensitive to neutralino masses up to several $\mathrm{TeV}$, which may be out of reach of accelerator experiments for some time to come.

\section{The Experimental Situation}

\subsection{Detection Techniques: $\gamma$-rays and Cosmic Rays}

As shown in Figure 5.1, $\gamma$-rays and cosmic rays span an enormous range of energies, from $1 \mathrm{MeV}$ to $10^{20} \mathrm{eV}$. Given this range, a single detection technique will not suffice. Satellite and balloon experiments above the Earth's atmosphere operate at MeV and $\mathrm{GeV}$ energies. At high energies, the particle flux is small enough so that space-borne instruments become flux limited. For $\gamma$-rays, the practical upper limit of sensitivity for the instruments on the Compton Gamma Ray Observatory is $\sim 20 \mathrm{GeV}$. For cosmic rays, the upper range of space-borne detectors is higher by several orders of magnitude due to the greater cosmic ray flux.

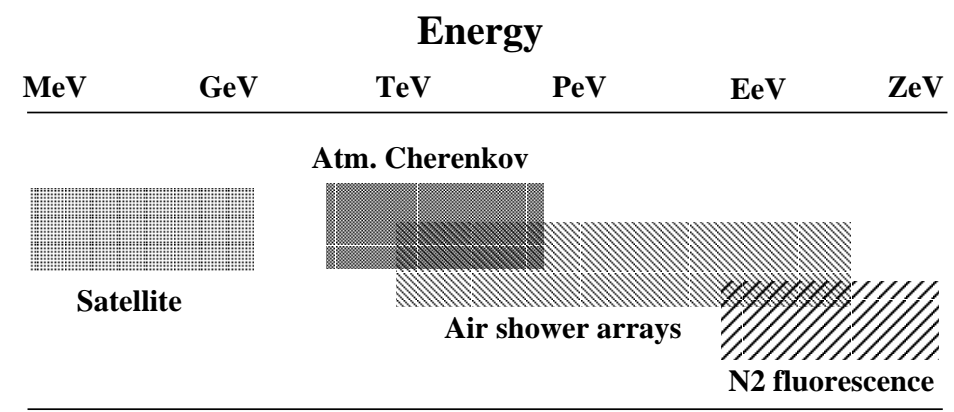

Figure 5: Detection techniques for high energy $\gamma$-ray and cosmic ray experiments. For each technique, an approximate energy range for current instruments is shown. For cosmic rays, the sensitivity of the satellite detectors extends to $\mathrm{TeV}$ energies.

At energies above the reach of balloon and satellite experiments, we use the Earth's atmosphere as the target and absorber of high energy particles. Primary $\gamma$-rays and cosmic rays interact in the atmosphere to create extensive air showers that propagate to the ground. Ground-based detectors sample the Cherenkov radiation, the 
charged particles, or the blue/UV fluorescence from nitrogen excitations to determine the arrival directions and energies of the incoming primary particles. As shown in Figure 5.1, atmospheric Cherenkov telescopes, air shower arrays, and Nitrogen fluorescence detectors operate in various energy ranges from $200 \mathrm{GeV}$ to $10^{20} \mathrm{eV}$.

\section{$5.2 \gamma$-ray Experiments and Results}

There are a number of ground-based $\gamma$-ray telescopes in operation around the world. This paper does not attempt to be comprehensive, and several recent articles have reviewed the experimental situation [10, 30]. The state-of-the-art Cherenkov telescopes include Whipple in Arizona, USA, HEGRA on the island of La Palma, CAT at Themis, France, and CANGAROO at Woomera, Australia. Operating air shower arrays include HEGRA and the Tibet Array in Yanbajing, Tibet.

$\gamma$-ray astronomy is a quickly changing field with a variety of sources and new phenomena discovered during the last decade. The EGRET experiment on the Compton Gamma Ray Observatory has detected $\sim 150$ sources at energies between $30 \mathrm{MeV}$ and $20 \mathrm{GeV}$. Many of the EGRET sources remain unidentified, but others have been associated with pulsars and AGN. At energies above $200 \mathrm{GeV}$, the current status of the field is shown in Figure 5.2. Atmospheric Cherenkov telescopes have detected at least seven VHE sources and five additional sources have been tentatively identified.

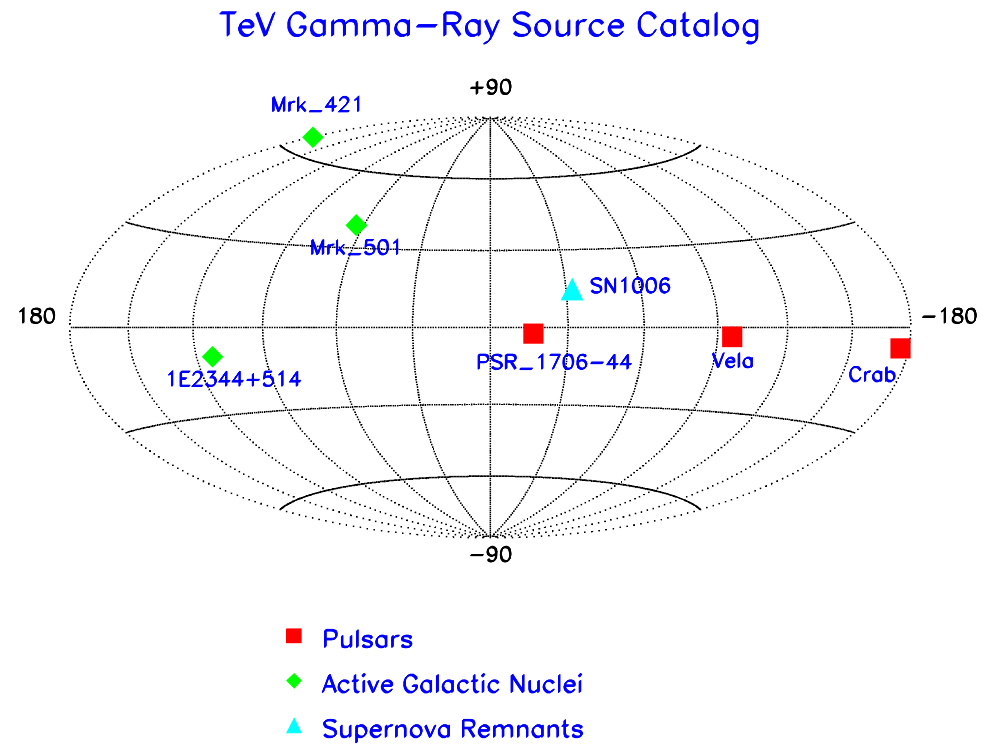

Figure 6: Current source map for the TeV $\gamma$-ray sky. The various source classifications are given by the legend. 
The sources detected at very high energies can be categorized as pulsar nebulae, AGN, and SNRs. The detection of each different source category was a significant advance which brought us new information about high energy astrophysical phenomena [11]. These detections have also raised numerous questions. For example, for AGN, remarkable emission has been detected from a few nearby sources, but many questions remain about how these sources work and why we cannot see more distant objects.

For the future, there are a variety of important areas that need exploration:

1. To date, no sensitive experiments have operated in the energy range between 20 and $200 \mathrm{GeV}$. New instruments, and possibly new techniques, are needed to explore this energy band where the likelihood of exciting new astrophysics is high. For example, it is in this energy range that we expect to measure spectral features resulting from the absorption of $\gamma$-rays from by the cosmic infrared radiation [31.

2. So far, it appears that we have detected very high energy radiation from the most luminous astrophysical sources. Significant increases in flux sensitivity should lead to the detection of fainter objects and more types of sources.

3. The most important $\gamma$-ray results at energies above $200 \mathrm{GeV}$ have come from atmospheric Cherenkov telescopes which have limited fields of view and operate only on dark, clear nights. We need instruments which have wide fields of view and operate with close to $100 \%$ duty cycle. Such instruments would be able to detect dramatic transient phenomena, such as gamma ray bursts.

To address the major goals for the future, there are a number of new high energy $\gamma$-ray telescopes soon to come on line or being proposed for the future. To extend the reach of ground-based telescopes to lower energies, the STACEE and CELESTE experiments each use large arrays of solar heliostat mirrors to detect fainter Cherenkov showers and thus to observe $\gamma$-rays at lower energies. Both experiments are in the final stages of construction and have reported promising initial results [32, 33]. The MAGIC imaging Cherenkov telescope [34, which consists of a large $17 \mathrm{~m}$ reflector and a state-of-the-art camera, is now under construction. MAGIC hopes to achieve an effective energy threshold as low as $20 \mathrm{GeV}$.

A new air shower experiment that has recently come on line is MILAGRO [35]. Consisting of a large man-made pond of purified water viewed by $\sim 1000$ photomultiplier tubes, MILAGRO detects air showers at a median energy threshold of $1 \mathrm{TeV}$. By using the air shower technique, MILAGRO will carry out the first all-sky survey at $\mathrm{TeV}$ energies. The encouraging initial results from a prototype of MILAGRO include the tentative detection of Mrk 501 and possible correlated $\mathrm{TeV} \gamma$-ray emission from a gamma ray burst [36. 
In order to substantially improve the sensitivity, and to extend the energy range, of the atmospheric Cherenkov technique, several groups are developing new telescopes that employ arrays of imaging reflectors [37]/ HESS, initially consisting of four $12 \mathrm{~m}$ reflectors in Namibia, and Super-CANGAROO, consisting of four $10 \mathrm{~m}$ reflectors located in Australia, will be the premier ground-based $\gamma$-ray telescopes in the Southern Hemisphere. In the Northern Hemisphere, VERITAS [39], is being proposed as an array of seven $10 \mathrm{~m}$ reflectors. As shown in Figure 5.2, the dramatic improvement in sensitivity of VERITAS over Whipple (which represents the current generation of atmospheric Cherenkov telescopes) will allow for much more detailed study of rapid very high energy phenomena.
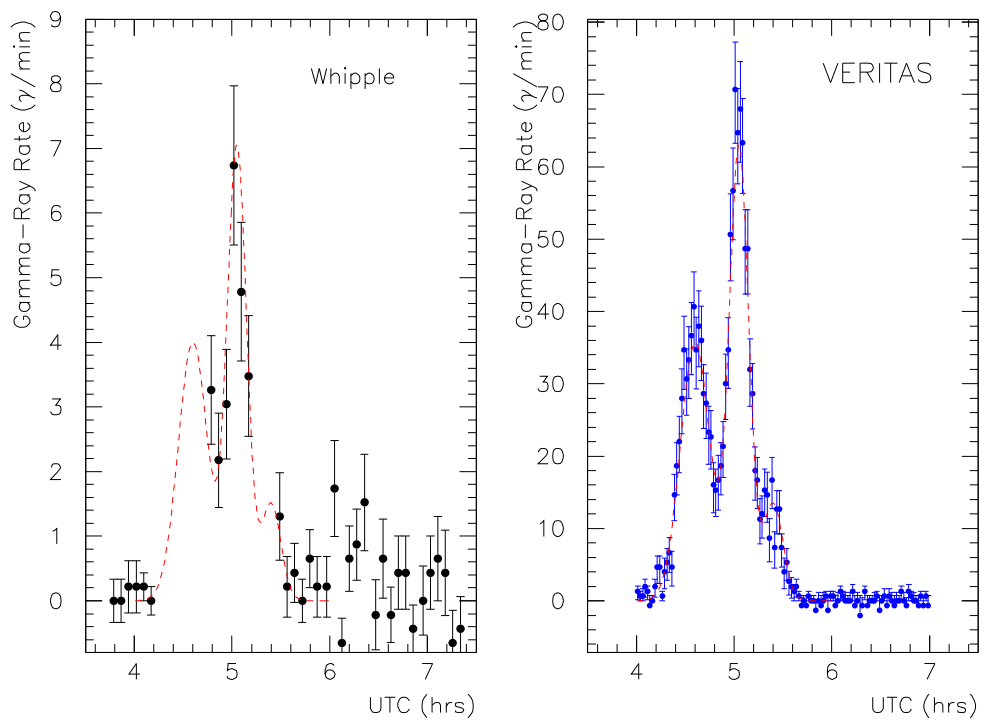

Figure 7: Left: response to a rapid AGN flare in 1996 by the Whipple Cherenkov telescope [38]. The dashed curve corresponds to a hypothetical intrinsic light curve consistent with the measured data. Right: simulated response of the VERITAS array to the same hypothetical light curve. VERITAS detects a much larger signal with much finer time resolution than Whipple.

The single most important new $\gamma$-ray telescope will be flown in space. GLAST [40], will be a state-of-the-art detector using many techniques of experimental particle physics, such as Si-strip tracking and CsI calorimetry. With a very wide field of view, and a suitable pointing strategy, GLAST will scan the entire sky on every orbit, offering unparalleled coverage of transient $\gamma$-ray phenomena, such as AGN flares and gamma ray bursts. GLAST will have substantially improved characteristics (angular resolution, energy resolution, energy range, etc.) relative to its predecessor, EGRET. The resulting improvement in sensitivity of GLAST will enable the detection of up 
to two orders of magnitude more sources (e.g. approximately 3000-4000 AGN). An artist's conception of GLAST is shown in Figure 5.2.

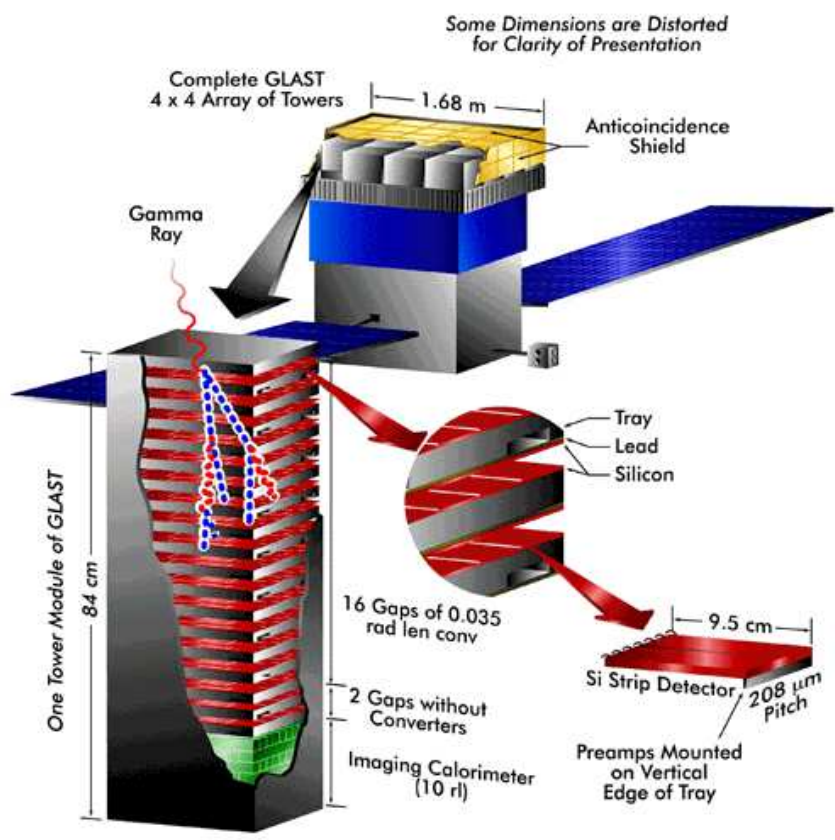

Figure 8: Artist's conception of GLAST, the next major satellite $\gamma$-ray telescope 40. The main GLAST instrument will consist of interleaved Si-strip/absorber layers for tracking and an imaging CsI calorimeter for energy and position measurement.

\subsection{Cosmic Ray Experiments and Results}

The variety of experimental techniques for the detection of high energy cosmic rays is summarized in Figure 5.1. Satellite instruments have measured the elemental composition of the cosmic rays at energies up to $10^{14} \mathrm{eV}$. An important outstanding issue concerns the level of antimatter, e.g. positrons, antiprotons, antihelium, etc. A primordial source of antimatter would be an important discovery. Searching for this source is one of the main goals of the Alpha Magnetic Spectrometer (AMS) satellite detector. In June 1998, AMS took initial cosmic ray data during a flight on the space shuttle Discovery. Initial results based on the AMS data have been presented [41]. AMS is now in the process of reconfiguration for an extended physics program on the International Space Station.

At energies above $10^{14} \mathrm{eV}$, ground-based air shower detectors provide the only effective way to carry out cosmic ray measurements. Determining the cosmic ray 
composition by indirect detection techniques has been notoriously difficult. No clear understanding of the composition exists in the energy region near the bend in the energy spectrum (the "knee" at $\sim 3 \times 10^{15} \mathrm{eV}$ ). It is in this region, however, where we expect to learn something about the origin of cosmic rays 42]. Future ground-based and satellite experiments may shed light on this difficult, but important, problem.

For the highest energy cosmic rays above the GZK cutoff, the current experimental situation is summarized in Figure 3.3. There is good evidence that the cosmic ray spectrum extends to $10^{20} \mathrm{eV}$ and beyond, but the statistics are not overwhelming. It is important to keep in mind that the flux of particles above $10^{20} \mathrm{eV}$ is approximately 1 per $\mathrm{km}^{2}$ per century! The AGASA experiment, which has detected the majority of the events beyond the GZK cutoff, has a collection factor of approximately 120 $\mathrm{km}^{2} \cdot \mathrm{sr}$. The newly commissioned Fly's Eye HiRes experiment improves upon this factor by an order of magnitude. Fly's Eye HiRes consists of two fluorescence detector sites located $13 \mathrm{~km}$ apart. Each detector employs spherical mirrors to reflect air shower fluorescence light onto photomultiplier tube cameras. The construction for the experiment has been completed, and preliminary results based on data-taking with an initial configuration have been reported [43]. The early Fly's Eye results provide clear confirmation of the AGASA result that the cosmic ray spectrum continues to $10^{20} \mathrm{eV}$, and beyond.

The exciting results relating to the highest energy cosmic rays have prompted the development of new, and even larger, cosmic ray experiments. The Auger Project plans to construct two giant air shower arrays, one each in the Southern and Northern Hemispheres. The arrays incorporates both particle and nitrogen fluorescence detectors [44]. Each array consists of 1600 particle detection stations on a grid covering approximately $3000 \mathrm{~km}^{2}$. Three fluorescence detectors are used to improve the energy calibration of the ground array. As shown in Figure 5.3, a detector station employs a large water tank viewed by self-contained instrumentation to record the arrival of the air shower particles. The Southern Hemisphere array is currently under construction in Mendoza, Argentina.

The Northern Hemisphere array of Auger is planned for construction in Millard County, Utah. A very large fluorescence detector, the Telescope Array, is also being considered for construction in the same general region as Auger. A possible future satellite experiment, OWL/Airwatch [45], would detect giant air showers using a downward-looking fluorescence detector. This ambitious instrument is currently under development by groups in the United States and Italy.

\subsection{Very High Energy Neutrino Astrophysics}

As pointed out by Greisen forty years ago 46], there is an obvious connection between $\gamma$-ray and neutrino astronomy. Both fields involve the detection of neutral particles produced as secondaries in high energy astrophysical accelerators. In many cases, the 


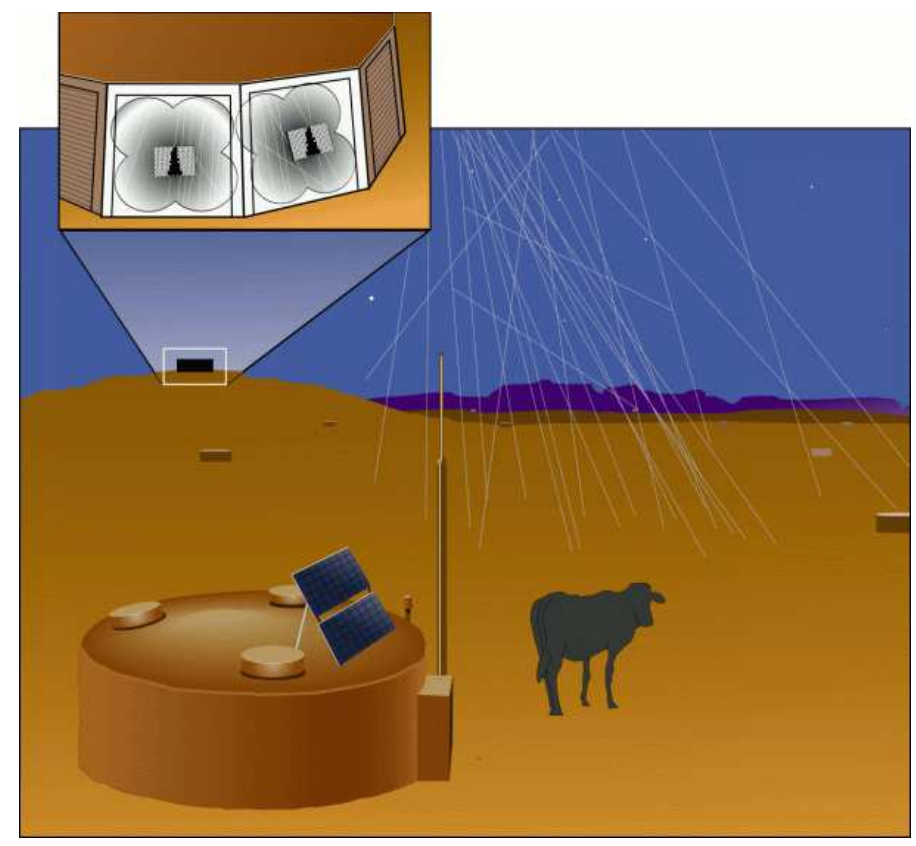

Figure 9: Artist's conception of the Auger Project for the detection of the highest energy cosmic rays [44. The water tank in the foreground is one of 1600 such tanks used to detect the air shower particles. A nitrogen fluorescence detector (one of three) is shown in the background. Two Auger sites are evisioned, one in each of the Northern and Southern Hemispheres.

same sources give rise to fluxes of high energy $\gamma$-rays and neutrinos.

There are important differences, however, between photon and neutrino astronomy. Neutrinos have the great advantage over $\gamma$-rays in that their very small interaction cross section allows them to travel unimpeded from their production sites to Earth. Thus, in principle, neutrino astronomy allows us to probe the dense central regions of objects such as gamma ray bursts, AGN, and supernovae. We can also search for neutrino signals coming from the annihilation of dark matter concentrated at the center of the Earth and Sun. Neutrino astronomy may also shed light on important aspects of particle physics. A clear demonstration of this possibility is the evidence for the oscillation of neutrinos produced by cosmic ray interactions in our atmosphere.

The most probable source of high energy astrophysical neutrinos is from the decay of charged pions produced in a hadronic beam. Thus, unlike $\gamma$-rays, which can arise from electromagnetic processes such as synchrotron radiation and inverse-Compton scattering, neutrinos will likely arise from astrophysical sources containing proton 
beams. Although we have not yet conclusively identified sources such as these, we know from the flux of cosmic rays that energetic hadrons are produced somewhere in large quantities.

The fact that neutrinos have a very small interaction cross section also poses a big difficulty for the experimentalist in that very large detectors are required. So far, this difficulty has limited the astrophysical information learned from neutrino telescopes. It is difficult to predict for certain how large experiments must be in order to detect neutrinos from AGN or gamma ray bursts. The appropriate detector collection area is probably $1 \mathrm{~km}^{2}$, or perhaps even larger [47].

For some time, it has been recognized that clear water (liquid or ice) would make a very suitable medium for neutrino detection. The basic idea is to instrument a large volume of water with photomultiplier tubes arranged on long strings. Upwardgoing neutrinos (coming from astrophysical sources on the other side of the Earth) interact in the large volume of Earth below the detector. The high energy muons produced from these interactions are detected via their Cherenkov radiation in the instrumented volume. The general method is shown in Figure 5.4. The upward-going signature is required to reduce the very large $\left(>10^{5}\right)$ background of downward-going muons produced in cosmic ray air showers. The cosmic rays also produce a flux of atmospheric neutrinos on the other side of the globe that can be used as a calibration source for the detector.

Early pioneering work in building very large neutrino telescopes was carried out by the DUMAND and BAIKAL collaborations. Indeed, BAIKAL was the first detector to observe very high energy atmospheric neutrinos 48]. Presently, the AMANDA installation at the South Pole represents the current state-of-the-art in high energy neutrino telescopes. AMANDA has grown in stages during the last five years. The current instrument, AMANDA-II, comprises 675 optical modules on 19 strings located at ice depths of 1450 to 2050 meters. AMANDA-II has a collection area of approximately 30,000 $\mathrm{m}^{2}$ ( $1 \mathrm{TeV}$ ). An earlier version of the detector, AMANDA-B, operated between 1997 and 1999 and recorded a large data set. As shown in Figure 5.4, by means of stringent quality cuts on the track fitting, a clean sample of 17 muon tracks due to atmospheric neutrino events has been isolated [49]. This result demonstrates that AMANDA can detect a neutrino signal.

To increase the likelihood of detecting astrophysical neutrinos, several groups have proposed water Cherenkov telescopes on much larger scales than AMANDA. There are three projects being considered for deployment in the deep Mediterranean water: ANTARES [50], NESTOR [51], and NEMO. Of these various efforts, the French ANTARES project is perhaps the furthest along. The ANTARES group has secured approval to build a telescope consisting of 1000 optical modules arranged on 13 vertical strings. The modules will deployed at water depths of 2100 to $2400 \mathrm{~m}, 30 \mathrm{~km}$ off shore. The baseline design for ANTARES calls for an effective collection area of approximately $0.1 \mathrm{~km}^{2}$. The neutrino energy threshold should be below $100 \mathrm{GeV}$ 


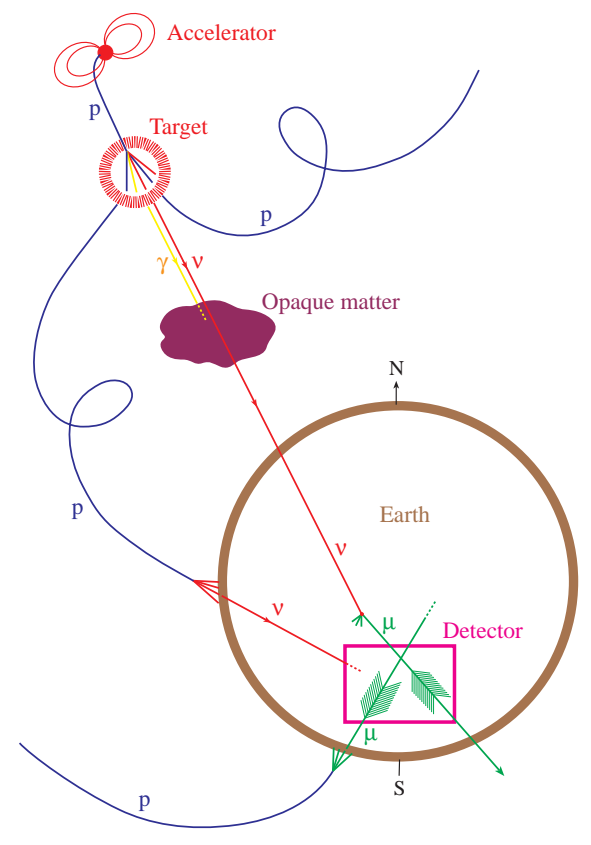

Figure 10: Detection technique for high energy neutrino telescopes [13]. Astrophysical or atmospheric neutrinos penetrate the Earth to produce upward going muons in the telescope. The telescope consists of strings of photomultiplier tubes that detect the Cherenkov radiation produced by the relativistic muon. The detector medium could be ice, as in the case of AMANDA, or deep sea water, as in the case of ANTARES.

which will permit the study of atmospheric neutrino oscillations, in addition to the search for neutrinos from astrophysical sources.

A very large $\mathrm{km}^{3}$ size detector is being considered for deployment in the South Pole ice. The IceCube collaboration is proposing an array of 81 detector strings arranged on a square grid of $125 \mathrm{~m}$ string spacing [52]. Each string would hold 60 optical modules with a vertical module spacing of $16 \mathrm{~m}$. The effective neutrino energy for IceCube of approximately $400 \mathrm{GeV}$ would be somewhat higher than for ANTARES, but IceCube will have a very large collection area $\left(1 \mathrm{~km}^{2} 10 \mathrm{TeV}\right)$ for neutrinos from astrophysical sources. 


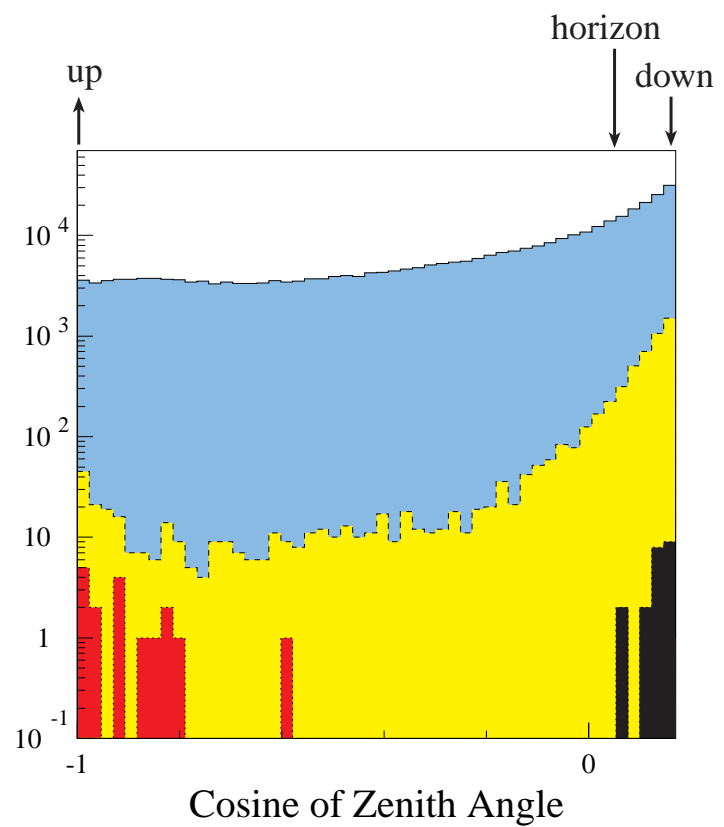

Figure 11: Zenith angle distribution for events detected by the AMANDA-B telescope [49]. The three different shadings indicate the distribution after successive quality cuts are applied to the data. The events in the dark shaded histogram near $\cos (\theta)=-1$ correspond to 17 upward-going muon tracks produced by atmospheric neutrinos.

\section{Summary}

Astronomy using very high energy particles ( $\gamma$-rays, cosmic rays, and neutrinos) is a diverse and rapidly developing field. It is currently a field largely driven by experimental results where significant scientific progress can be made on a relatively short time scale. For example, in the last few years there have been several exciting discoveries. Using $\gamma$-rays, we are probing remarkable and unexpected phenomena in objects such as active galaxies and gamma ray bursts. We are also searching for the origins of the cosmic radiation. At the very highest energies, we are discovering cosmic ray particles that probably should not be there, but are. Discoveries in this field often raise as many questions as they answer.

For the future, there will be an expanding interest in this field, both to understand astrophysics under extreme conditions and to search for evidence of physics beyond the standard models of elementary particles and cosmology. Future next-generation experiments in space and on the ground will greatly expand our discovery horizon. For $\gamma$-rays, the new projects include more powerful atmospheric Cherenkov telescopes and a new $\gamma$-ray satellite, GLAST. Larger air shower detectors, such as Fly's Eye HiRes 
and Auger, will explore questions relating to the highest energy cosmic rays. In the world of neutrinos, the AMANDA experiment has demonstrated the ability to detect neutrinos produced in the atmosphere. Future experiments, such as IceCube and

ANTARES, will greatly expand the possibility of detecting high energy astrophysical neutrino sources. We can only hope that our overall knowledge of high energy particles from the Universe advances at the same rate as new instruments are being constructed.

I wish to acknowledge the help and encouragement of many people in the particle physics and astrophysics communities. The contributions of the following people were particularly important: Katsushi Arisaka, Steve Barwick, Michael Catanese, Corbin Covault, Jim Cronin, Francis Halzen, Charles Jui, Tadashi Kifune, Peter Leonard, Eckart Lorenz, John Matthews, Masaki Mori, Gus Sinnis, David Smith, Simon Swordy, Steve Ritz, Pierre Sokolsky, Masahiro Takeda, Masahiro Teshima, Trevor Weekes, and Heinz Völk. I also thank the organizers of the Lepton-Photon Symposium (especially John Jaros, Helen Quinn, and Michael Peskin) for their encouragement and patience. Any inaccuracies are my fault alone. This research is supported in part by the National Science Foundation.

\section{References}

[1] M. Harwit, Cosmic Discovery, MIT Press, Cambridge MA, 1984.

[2] See, for example: E. Waxman, Phys. Rev. Lett. 75, 386 (1995).

[3] See, for example: F. Halzen and D.W. Hooper, Astrophys. J. 527, L93 (1999).

[4] W.S. Paciesas et al., Astrophys. J. Suppl. 122, 465 (1999).

[5] See, for example, Astron. Astrophys. Suppl. 138, pps. 395-607 (1999).

[6] P. Meszaros and M. Rees, Astrophys. J. 476, 232 (1997).

[7] A.I. MacFadyen and S.E. Woosley, Astrophys. J. 524, 262 (1999).

[8] R.J. Nemiroff et al., Astrophys. J. 435, L133 (1994).

[9] C. Akerlof et al., Nature 398, 400 (1999).

[10] R.A. Ong, Phys. Rep. 305, 93-202 (1998).

[11] M. Catanese and T.C. Weekes, Proc. Astron. Soc. Pacific 111, 1193 (1999).

[12] J. Quinn et al., Astrophys. J. 518, 693 (1999).

[13] F. Halzen, private communication. 
[14] K. Greisen, Phys. Rev. Lett. 16, 748 (1966); G.T. Zatsepin and V.A. Kuz'min, Zh. Eksp. Teor. Fiz. 4, 114 (1966).

[15] M. Takeda et al., Phys. Rev. Lett. 81 (1998) 1163; AGASA Collaboration, Proc. 26th. Int. Cosmic Ray Conf. (Salt Lake City), 3, 252 (1999).

[16] D.J. Bird et al., Astrophys. J. 441, 114 (1995).

[17] O.C. de Jager et al., Astrophys. J. 457, 253 (1996).

[18] E. Fermi, Phys. Rev. 75, 1169 (1949).

[19] P.O. Legage and C.J. Cesarsky, Astron. Astrophys. 125, 239 (1983).

[20] A.M. Hillas, Ann. Rev. Astron. Astrophys. 22, 1 (1984).

[21] See, for example: L. Maraschi, G. Ghisellini, and A. Celotti, Astrophys. J. 397, L5 (1992).

[22] C.D. Dermer, R. Schlickheiser, and A. Mastichiadis, Astron. Astrophy. 256, L27 (1992); M. Sikora, M.C. Begelman, and M.J. Rees, Astrophys. J. 421, 153 (1994).

[23] K. Mannheim, Astron. Astrophys. 269, 67 (1993).

[24] B. Bhattacharjee, C.T. Hill, and D.N. Schramm, Phys. Rev. Lett. 69, 567 (1992); R.J. Protheroe and T. Stanev, Phys. Rev. Lett. 77, 3708 (1996).

[25] S.W. Hawking, Nature 248, 30 (1974).

[26] F. Halzen, E. Zas, J.H. MacGibbob, and T.C. Weekes, Nature 353, 807 (1991).

[27] I.F.M. Albuquerque, G.R. Farrar, and E.W. Kolb, Phys. Rev. D59, 015021 (1999).

[28] G. Jungman, M. Kamionkowski, and K. Griest, Phys. Rep. 267, 195 (1996).

[29] L. Bergstrom, P. Ullio, and J.H. Buckley, Astropart. Phys. 9, 137 (1998).

[30] C.M. Hoffman, C. Sinnis, P. Fleury, and M. Punch, Rev. Mod. Phys. 71, 897 (1999).

[31] F.W. Stecker, Astropart. Phys. 11, 83 (1999). J.R. Primack et al., Astropart. Phys. 11, 93 (1993).

[32] S. Oser et al. Proc. 26th Int. Cosmic Ray Conf. (Salt Lake City), 3, 464 (1999).

[33] M. de Naurois, Proc. 26th Int. Cosmic Ray Conf. (Salt Lake City), 5, 211 (1999). 
[34] J.A. Barrio et al. The Magic Telescope Design Study, MPI-PhE/98-5 (1998).

[35] C. Sinnis et al., Nucl. Phys. B. (Proc. Suppl.) 43, 131 (1995).

[36] R. Atkins et al., Astrophys. J. 525, L25 (1999); R. Atkins et al., astro-ph 0001111, to be published in Astrophys. J. Lett.

[37] See talks by W. Hofmann and T. Kifune in Proc. Towards a Major Atmospheric Cherenkov Telescope VI (Snowbird), in press (1999).

[38] J.A. Gaidos et al., Nature 383, 319 (1996).

[39] F. Krennrich, Proc. Towards a Major Atmospheric Cherenkov Telescope VI (Snowbird), in press (1999).

[40] N. Gehrels and P. Michelson, Astropart. Phys. 11, 277 (1999).

[41] J. Alcaraz et al., Phys. Lett. B461, 387 (1999); J. Alcaraz et al., Phys. Lett. B472, 215 (2000).

[42] A.A. Watson, Proc. 25th Int. Cosmic Ray Conf. (Durban), ed. M.S. Potgieter et al. (World Scientific, Singapore), 8, 257 (1997).

[43] C.C.H. Jui, Proc. 26th Int. Cosmic Ray Conf. (Salt Lake City), Invited, Rapporteur, and Highlight Papers, in press (2000).

[44] M. Boratav, Proc. 25th Int. Cosmic Ray Conf. (Durban), ed. M.S. Potgieter et al. (World Scientific, Singapore), 5, 205 (1997).

[45] L. Scarsi, Proc. 26th Int. Cosmic Ray Conf. (Salt Lake City), 2, 348 (1999).

[46] K. Greisen, Ann. Rev. Nucl. Sci. 10, 63 (1960).

[47] T.K. Gaisser, F. Halzen, and T. Stanev, Phys. Rep. 258, 173 (1995).

[48] V.A. Balkanov et al., Astropart. Phys. 12, 75 (1999).

[49] E. Andres et al., Astropart. Phys. 13, 1 (2000). E. Andres et al., Proc. 17th Int. Workshop on Weak Interactions and Neutrinos (Cape Town), astro-ph/9904379 (unpublished).

[50] L. Moscoso, Nucl. Instrum. Meth. A409, 454 (1998); see also the proposal available at: http://antares.in2p3.fr/antares/.

[51] L. Trasatti, Nucl.Phys. (Proc.Suppl.) 70, 442 (1999).

[52] E. Andres et al., Proc. 26th Int. Cosmic Ray Conf. (Salt Lake City), 2, 428 (1999). 